\title{
О выборе форматов данных для электронных мультимедийных коллекций
}

\author{
Н.В. Борисов, В.В. Захаркина, И.А. Мбого, П.П. Щербаков \\ Санкт-Петербургский государственный университет \\ n.borisov@spbu.ru, v.zaharkina@spbu.ru, i.mbogo@spbu.ru, \\ p.scherbakovespbu.ru
}

\section{Аннотация}

Разрабатывается система, позволяющая обеспечить полноценный доступ к мультимедийным коллекциям через веб интерфейс без установки и использования дополнительных приложений или плагинов на стороне клиента. В качестве одной из сфер приложения рассматривается поддержка электронного мультимедийного журнала. Проанализированы требования, диктуемые особенностями хранения, доступа и представления мультимедийных элементов электронных коллекций со стороны службы поддержки коллекции, владельцев контента и внешних пользователей. В статье рассмотрены основания для включения или отказа от поддержки тех или иных форматов хранения элементов коллекции на примере результатов, полученных при разработке инструментальной платформы электронного научного журнала, обеспечивающей публикацию мультимедийных материалов через веб-интерфейс. Система публикации мультимедийных коллекций, поддерживающая, на настоящий момент, ограниченный набор форматов, доведена до уровня развертывания «из коробки» как в варианте универсальном, так и в варианте электронного мультимедийного журнала. Предполагается в дальнейшем осуществить поддержку всех обсужденных в статье форматов.

Ключевые слова: цифровые коллекции, мультимедиа контент, форматы данных

Библиографическая ссылка: Борисов Н.В., Захаркина В.В., Мбого И.А., Щербаков П.П. О выборе форматов данных для электронных мультимедийных коллекций // Информационное общество: образование, наука, культура и технологии будущего. Выпуск 4 (Труды XXIII Международной объединенной научной конференции «Интернет и современное общество», IMS-2020 (сборник научных статей). - СПб: Университет ИТМО, 2020. С. 58-71. DOI: $10.17586 / 2587-8557-2020-4-58-71$

\section{1. Открытые системы для поддержки электронных коллекций}

На протяжении последних нескольких десятков лет происходит расширение сети интернет и ее веб-сегмента. Увеличивается не только аудитория, но и способы использования данных, размещенных в сети. Расширение числа информационных сервисов, появление социальных сетей сделало доступ к информации через интернет обычным явлением. Оцифровка большого числа объектов культуры, науки потенциально позволяет мгновенно получить к ним доступ, вне зависимости от их физического расположения. В этих условиях возрастает роль оформления тематических собраний цифровых ресурсов с поддержкой эффективных способов доступа к ним - электронных коллекций. В свободном доступе появляются сервисы, позволяющие в той или иной степени решать подобные задачи. Общим свойством подобного рода сервисов является то, что от посетителя такой коллекции практически не требуется приложения никаких усилий 
для доступа, в том числе финансовых затрат. Единственными требованиями, в идеале, являются наличие на устройстве пользователя стандартного браузера и доступ к сети. Обратной стороной использования таких сервисов является делегирование прав на использование данных держателям сервисов и, возможно, согласие на просмотр рекламы. В некоторых случаях такое положение дел может оказаться неприемлемым, и создатели электронных коллекций могут попытаться реализовать собственную версию платформы со специфическим функционалом, однако, неприемлемыми могут оказаться и трудозатраты на создание и сопровождение таких сервисов.

Использование веб-публикаций становится все более актуальным и в научной сфере. Для обеспечения долгосрочного хранения информационных ресурсов, обмена метаинформацией и др. создан ряд ИС, используемых научным и образовательными сообществами. Приведем несколько примеров систем, используемых для электронных научных публикаций:

- Система поддержки цифровых хранилищ (институциональных репозиториев) широко используются для построения архивов открытого доступа и электронных библиотек, позволяющих создавать, хранить и распространять цифровые материалы. К ним относятся такие открытые программные платформы как DSpace, EPrints, GreenStone, Fedora и другие. Наиболее авторитетные платформы поддерживаются крупными научными и технологическими организациями, так платформа DSpace разрабатывалась совместно компанией HewlettPackard и библиотеками MIT (Massachusetts Institute of Technology) $[1,2]$.

- Некоторые электронные научные журналы используют электронные издательские системы, такие как Open Journal Systems (OJS). Основными достоинствами такого подхода является реализация полного издательского цикла для подготовки электронных публикаций. Следует отметить, что верстка проводится вне системы, а в OJS загружаются уже готовые файлы. В этой системе не предусмотрена реализация отображения мультимедийного контента. При этом формат научной публикации остается традиционным, а в веб она может быть представлена в двух форматах - PDF и статический HTML.

- Вопросы интеграции решаются на базе технологии обмена метаданными, основанной на протоколе ОАІ-PMН.

- Развивается концепция «живых публикаций». Живая публикация - размещенная в интернете в свободном доступе научная работа, которая постоянно поддерживается ее автором в актуальном состоянии [3].

- Начиная с 2014, несколько журналов издательства Elsevier, начали публиковать отзывы рецензентов вместе со статьей. Такой подход, называемый открытым рецензированием (Open review) приводит к улучшению качества статей, признается вклад рецензента в публикационный процесс [4].

С начала 2000-х годов активно начинают развиваться такие сетевые электронные ресурсы как электронные журналы. Электронные журналы публикуют статьи как в формате PDF, так и в HTML, который предоставляет больше возможностей для публикации, в том числе и мультимедийных материалов.

Ограничиваясь анализом журналов научной направленности можно представить следующие выводы:

- приём редакцией текстов статей и сопутствующих мультимедийных материалов осуществляется посредством электронной почты. Т.е. процесс загрузки элементов коллекции нетехнологичен;

- редакции просят указывать место в тексте статьи, куда необходимо вставить соответствующий мультимедийный материал. Т.е. авторы непосредственно не принимают участие в вёрстке статьи;

- ни один из проанализированных журналов не публикует полный спектр мультимедийных файлов. Основными публикуемыми форматами являются: 
графические файлы различных форматов (например, JPG, GIF, PNG), видео (например, AVI, MPG, MOV), анимация (SWF, GIF) [5], аудио различных форматов, презентации. Есть отдельные журналы, публикующие кроме всего перечисленного спектра мультимедийных форматов виртуальную реальность в формате VRML (журнал «Научная визуализация», http://sv-journal.org/). T.e. нет системы, поддерживающей относительно широкий спектр мультимедийных форматов;

- для отображения видео в статьях используются различные видео проигрыватели или встраиваются видео, размещённые в соответствующих облачных видео сервисах. Т.е. для отображения элементов коллекции используются сторонние приложения, поддержка которых в дальнейшем не гарантирована;

- сайты журналов либо являются оригинальными разработками, либо представлены на платформах издательств. Наибольший спектр отображаемых мультимедийных материалов публикует журнал «Научная визуализация» (http://sv-journal.org.). Однако, даже на страницах этого журнала для просмотра некоторых мультимедийных объектов (например, 3d, http://sv-journal.org./example/index.html) требуется использование дополнительного программного обеспечения, установленного на компьютер пользователя. Т.е. для представления элементов коллекции пользователь должен использовать дополнительные приложения или плагины.

С преодолением ряда подобных проблем авторы столкнулись при разработке инструментальной платформы электронного научного журнала, обеспечивающей публикацию мультимедийных материалов и доступ к ним через веб-интерфейс [6, 7]. Одной из таких проблем и является выбор форматов данных, которые могут прозрачно обрабатываться в рамках платформы и, по возможности, обеспечивать широкие возможности авторам для публикации мультимедийных данных.

Не претендуя на исчерпывающее описание данных, которые могут храниться в широком спектре разнообразных коллекций, обратимся к спектру мультимедийных форматов, актуальных для статей научных онлайн-журналов. Приведенный список основных типов мультимедийных компонентов, в дальнейшем может расширяться с развитием платформы, однако даже при использовании рассмотренного перечня типов, практически любой контент может быть представлен адекватно.

Основными предпосылками, которые рассматривались при отборе и анализе поддерживаемых типов, данных были следующие:

- предоставляемые авторами материалы должны эффективно обрабатываться на сервере, причем от сотрудников не должно требоваться большого объема рутинных действий и виртуозное владение современными программными средами разработки. Так для правильного воспроизведения сложного объекта может быть необходимо сохранить структуру проекта, содержащего десятки каталогов и сотни, может быть, и тысячи отдельных файлов, причем в проекте могут сохраняться абсолютные пути к используемым ресурсам, так что даже при копировании всего дерева проекта в другое место правильное воспроизведение объекта может нарушиться, а встраивание такого объекта в документ может потребовать от сотрудников журнала (команды программнотехнической поддержки коллекции) применения высокопрофессиональных навыков в различных предметных областях;

- пользователь должен иметь доступ до статьи и отдельных ее компонентов (отдельных элементов коллекции) без установки специализированного программного обеспечения. В идеале, пользователю должно быть достаточно выхода в сеть интернет и установленного на его компьютере стандартного браузера. Использование авторами специальных, иногда проприетарных, расширений может привести к тому, что на компьютере пользователя-читателя (внешнего пользователя, пытающегося получить доступ к элементам коллекции) воспроизведение объекта окажется невозможным.

- выбранный ограниченный набор поддерживаемых форматов должен, по возможности, обеспечивать широкие возможности использования мультимедийных ресурсов. 
В настоящий момент к типам поддерживаемых мультимедийных компонентов и типам, которые будут поддерживаться в перспективе, можно отнести следующие:

- текстовые фрагменты, количественные данные;

- растровые и векторные изображения;

- аудиоданные;

- видео различных форматов, в том числе и формата Видео360;

- 3D-модели;

- сцены виртуальной и дополненной реальности;

- картографические данные;

- диаграммы;

- формулы и другие.

Наряду с представлением мультимедийных объектов важным аспектом является и способ хранения.

Следует отметить, что элементом мультимедийной коллекции может быть произвольный простой фрагмент данных, допустимых для платформы типов, но и произвольный агрегат, включающий фрагменты данных разных типов.

\section{2. Аспекты подготовки мультимедийных материалов для онлайн- журналов. Три формата и три контекста}

По существу, для каждого мультимедийного элемента онлайн-статьи актуальны, как минимум, три формата:

- формат, предназначенный для конечного пользователя, просматривающего онлайнстатью в браузере;

- формат внутреннего хранения;

- формат, в котором автор статьи предоставляет материалы в редакцию.

Рассматривая вопросы, связанные с форматами мультимедийных элементов онлайнстатей, мы учитываем три контекста:

- эффективность работы пользователей, представляющих различные роли;

- аспекты технологической реализации и возможности сопровождения на разных стадиях жизненного цикла информационной системы;

- обеспечение сохранения исходных данных без потерь, связанных с форматами и параметрами, актуальными на момент публикации.

Рискнём сказать, что третий из упомянутых контекстов весьма часто остаётся за границами рассмотрения при обсуждении текущих и перспективных технологических решений. Преобразование данных к актуальным форматам конечного представления в браузере может в дальнейшем обернуться потерями. Достаточно, например, обратиться к архивным версиям сайтов, созданных 10-15 лет назад. Изображения малых размеров и видео низкого качества (в формате SWF) специально готовились для публикации, но в большинстве случаев исходные данные уже утрачены.

Требования к форматам мультимедийных элементов в значительной степени определяются задачами и спецификой работы пользователей, представляющих различные роли в рамках информационной системы.

Конечный пользователь («читатель») должен иметь возможность адекватного просмотра мультимедийной статьи на широком спектре устройств. Воспроизведение мультимедийных элементов должно быть корректным на актуальных операционных системах и в современных браузерах. Более того, существенной тенденцией последнего десятилетия является стремление реализовать всю требуемую функциональность исключительно средствами браузера, без сторонних плагинов. Рискнём сказать, например, что в настоящее время предложение установить некий плагин для просмотра видео будет воспринято однозначно негативно. 
Автор использует для подготовки мультимедийных элементов привычные приложения или онлайн-сервисы. В результате он получает мультимедийные элементы будущей статьи в одном из форматов, экспорт в которые допускается приложением. Какой должна быть политика онлайн-журнала? Можно выделить несколько подходов, из которых здесь упомянем лишь два. Первый вариант предполагает жёсткое ограничение: от автора требуется подготовить мультимедийный элемент в одном из форматов, которые могут быть непосредственно отображены в браузере. Второй вариант допускает подачу автором материалов в одном из спектра форматов, подлежащих дальнейшему преобразованию. Преобразование может быть проведено либо вручную контент-менеджером, либо соответствующим программным модулем информационной системы.

Контент-менеджер загружает в систему подготовленный автором материал. Чем строже требования к форматам мультимедийных элементов, подготовленных автором, тем меньших усилий требуют технические моменты, связанные с публикацией. Минимальной квалификации от контент-менеджера требует ситуация, при которой необходимо лишь скопировать текст статьи в соответствующее поле и загрузить мультимедийные файлы без их предварительной обработки. При этом в нетривиальном случае усложняется работа автора, связанная с конвертированием исходных данных в требуемые форматы.

Отметим, что текущие представления об «актуальных ОС» и «современных браузерах» в недалёкой перспективе обретут абсолютно иной смысл, иными будут и устройства, каналы связи, стилистические тенденции, технологические возможности... Именно поэтому основной формат внутреннего хранения, как правило, предполагает определённую избыточность.

\section{3. Типы компонентов мультимедийных коллекций}

\section{1. Текстовые данные}

Наличие текстовых фрагментов в статье представляется очевидным. Используемые текстовые фрагменты могут быть как совсем небольшими - содержанием полей анкеты так и значительного размера. Использование текста подразумевает использование языка, который, в свою очередь, ассоциируется с возможным набором используемых символов алфавитом. Легко представить ситуацию, когда в одном текстовом фрагменте могут использоваться несколько языков, и соответственно, несколько наборов символов. Такая ситуация может иметь некоторые последствия. Например, при формировании списков, от владельцев коллекции может понадобиться соответствующие правила лексикографического упорядочивания. Возможны и другие нюансы в использовании текстовых фрагментов. В развиваемой платформе предполагается использование двух основных языков - русского и английского. В связи с этим не предполагается использование текстов с направлением текста «справа налево», как в арабских языках, или «сверху вниз», как в японском языке. С другой стороны, ряд символов в тексте, которые используются в некоторых предметных областях, может встречаться в представленных текстах, но в набор символов русского или латинского (и, кстати, греческого, часто используемого в математических формулах) наборов символов не входить. Кроме того, использование расширенного форматирования текста (курсив, полужирность, подчеркивание, размер и цвет текста и фона, использование различных шрифтов, структурных элементов, таких как заголовки, абзацы, и др.) подразумеваются как стандартные свойства текста.

Таким образом, для представления текстовых фрагментов может быть использован UNICODE позволяющий адекватно отображать широкий спектр символов, формат TXT для простых текстовых фрагментов и RTF (RichTextFormat) для сложных, как общедоступные и открытые. Возможностей их для представления текстовых фрагментов в журнальных статьях представляется достаточным. 
Примерно аналогичными с RTF возможностями может обладать текст, размеченный тегами HTML с привлечением таблиц стилей, однако использование имен, введенных автором разметки, может прийти в противоречие с разметкой, создаваемой при отображении элемента коллекции в браузере, что может привести к возникновению нежелательных сложностей при конвертации.

Хранение фрагментов текста в виде отдельных файлов или текстовых полей серверов баз данных проблем не представляет.

\section{2. Количественные данные}

Ряд характеристик элементов коллекций может выражаться количественными значениями. Более того, ряд характеристик, не имеющих явную количественную природу, при оцифровке могут выражаться количественными значениями, так цвет, наряду с текстовым описанием (например, красный - red), может описываться числовым значением палитры (например, \#FF0000).

Для хранения и представления количественных данных имеется достаточно возможностей, являющихся на настоящий момент стандартными. Однако, как и для текстовых фрагментов, для представления количественных данных могут использоваться различные формы - «арабское» или «римское» написание; двоичное, десятичное или шестнадцатиричное представление, в виде рациональных дробей, поэтому при поддержке платформы необходимо предусмотреть соответствующие возможности для хранения и отображения.

Для особых случаев, когда стандартных средств хранения или представления оказывается недостаточно (например, для преставления числа ПИ с точностью до сотого знака после десятичной точки), можно использовать представление в виде текстовых строк. В любом случае, количественные данные могут храниться в виде стандартных числовых полей или в текстовом представлении.

Для представления связанных групп чисел могут использоваться специальные объекты, такие как таблицы, графики, диаграммы, формулы.

\section{3. Растровые изображения}

С появлением цифровых камер широкого спектра, программных средств редактирования цифровых изображений, хранение, передача и отображение изображений стало столь же обычным и распространенным, как и текстовых данных. Вместе с распространением использования изображений расширилось число различных форматов хранения изображений, обеспечивающих особые возможности представления и использования

Растровые изображения характеризуются размером пиксельной матрицы - растром (width x height), глубиной цвета пикселя и форматом хранения. Первые характеристики определяют, как выглядит изображение при отображении без преобразования, формат хранения определяет действия, которые должны быть выполнены, прежде чем будет сформирована пиксельная матрица, которая будет отображаться. Размер отображаемой пиксельной матрицы может отличаться от непреобразованной, причем современные программные средства позволяют сделать такое преобразование настолько быстро, что у пользователей, которые не задумываются о технических аспектах хранения и отображения изображений, создается ложное впечатление об элементарности этих действий. Однако автоматическое преобразование исходного размера изображения к размеру, задаваемому характеристиками документа, в контекст которого помещается изображение, может приводить к некоторым негативным последствиям - при увеличении масштаба изображения может теряться четкость, при уменьшении могут теряться мелкие детали. Кроме того, передача на компьютер пользователя изображения более высокого разрешения, чем отображаемое, потребляет дополнительный сетевой трафик. 
Если в бытовых ситуациях такое положение дел может рассматриваться как удовлетворительное, а вносимые искажения несущественными, то в некоторых профессиональных областях, например, в медицине, такая ситуация представляется как неприемлемая.

Для решения специфических задач разрабатываются специальные форматы. Так многие производители фотокамер могут предоставлять изображение в формате RAW - в виде необработанных измерений непосредственно с сенсора, что позволяет осуществить расширенное редактирование фотографии, однако обрабатывать такие файлы можно только с использованием специальных программ, причем для разных производителей программы обычно несовместимы. Разработчики новых графических растровых форматов, таких как JPEG200, WebP, BPG, WBMP, FLIF демонстрируют преимущества, которые дает их использование, однако пока отсутствует поддержка их современными браузерами, а в некоторых случаях может потребоваться и специальная лицензия. Исходя из сложившейся на настоящий момент ситуации, принято решение на настоящем этапе использовать в коллекциях только графические растровые форматы, поддерживаемые непосредственно браузерами, так как в статьях изображения имеют иллюстративный, а не документальный характер.

Формат - для браузеров стандартными форматами растровой графики являются форматы JPG (Joint Photogrph expert Group, возможны и другие суффиксы-расширения в названии файла - .jpeg, .jpe), PNG (Portable Network Graphics) и GIF (Graphic Interchange Format), которые автоматически распознаются и обрабатываются браузерам и являются открытыми стандартами.

Таким образом, можно сформулировать следующие рекомендации для представления растровых изображений:

- формат изображения JPG, PNG или GIF.

- размер изображения - соответствует размеру изображения, отображаемому на компьютере пользователя.

Особенно важным является согласование размеров изображения для группы, которая загружается в "слайдер" или "галерею"

\section{4. Векторная графика}

В некоторых ситуациях вместо растровых изображений могут использоваться векторные. В этом случае для хранения изображения используется не матрица пикселов, а описание алгоритма его построения. Для таких изображений масштабирование без потери качества может осуществляться в несоизмеримо больших границах, чем для растровых. Наиболее известными векторными графическими форматами являются WMF (Windows Meta File), CDR (CorelDRaw Document), AI (Adobe Illustrator), EPS(Encapsulated PostScript), однако непосредственная поддержка в браузере этих форматов оставляет желать лучшего. В качестве векторного формата для графических файлов предполагается использовать SVG (Scalable Vector Graphics — масштабируемая векторная графика), продвигаемый консорциумом W3C и имеющий поддержку во всех распространенных браузерах.

\section{5. Аудио}

Аудио фрагменты так же представляются компонентом, который должен поддерживаться в мультимедийной статье. Аудиофайлы характеризуются следующими характеристиками форматом файла-контейнера, кодеком - алгоритмом компрессии аудиоданных, частотой дискретизации по времени и амплитуде, числом каналов воспроизведения. В настоящий момент имеется несколько форматов файла-контейнера .WAV, .AAC, .OGG, .MP3, .M4A, .AIFF, .FLAC и другие. Как правило, формат файлаконтейнера определяет кодек, которым осуществлено сжатие хранимых данных. Исходя из требования широкой доступности, следует учитывать, какие форматы аудиоданных 
воспроизводятся без дополнительных действий пользователя. Так на платформе WINDOWS в стандартном дистрибутиве устанавливаются кодеки, соответствующие контейнерам .WAV, .MP3, .WMA в iOS - .MP3, M4A, .AIFF, .WAV, в LINUX (в том числе и для OS ANDROID) - .WAV, .OGG, .MP3. Кроме того, подавляющее число компьютеров оснащены двухканальными акустическими системами, так что, например, звук в формате DOLBY SURROUND 7.1 на них воспроизводиться не будет. Еще одним аспектом, который следует учитывать - длительность аудио фрагмента. Для фрагмента, длительность которого ограничивается несколькими секундами, содержащий его файл можно хранить непосредственно на сервере и передавать пользователю по прямой ссылке. Если длительность фрагмента в районе минуты и больше - то следует использовать потоковую передачу, что может потребовать поддержки отдельного потокового сервера. В подобной ситуации возможно использование внешнего хранения файлов на медиа серверах. В этом случае файл размещается на специальном медиа сервере (например YouTube.com) и от службы сопровождения сервера получается ссылка на доступ к ресурсу и, если нужно, фрагмент программного кода, который можно встроить в веб-документ, позволяющий получить из веб-документа доступ к ресурсу и обеспечить выполнение ряда условий. В случае использования подобной схемы хранения медиа-сервер при взаимодействии с пользователем анализирует состав программного и аппаратного обеспечения и передает данные в подходящем формате, даже если на сервер они помещались в другом формате.

Таким образом, можно сформулировать следующие рекомендации для представления аудиофайлов:

- формат файла-контейнера - MP3, WAV, OGG (браузеры, поддерживающие стандарт HTML5, могут их воспроизводить самостоятельно), отдельно можно представлять файлы в формате MIDI, поддержка которого было введена в браузерах еще на уровне HTML 3.2;

- число каналов 1 или 2;

- частоты дискретизации по амплитуде и времени имеет смысл выбирать из стандартных распространенных (22 050 Гц, 32000 Гц, 44100 Гц, 48000 Гц, глубина дискретизации до 16 бит) значений на усмотрение автора (более высокие параметры имеет смысл использовать при специфических коллекциях).

\section{6. Видео}

Видео файлы так же представляются компонентом, который должен поддерживаться в мультимедийной статье. Видеофайлы характеризуются следующими характеристиками форматом файла-контейнера, кодеком - алгоритмом компрессии аудиоданных и видеоданных, частотой кадров и их размером. В настоящий момент имеется несколько форматов файла-контейнера - .AVI, .WMV, .FLV, .MP4, .MPEG, .MOV, .MKV и другие. Как правило, формат файла-контейнера определяет аудио и видео кодеки, которыми осуществлено сжатие хранимых данных. Для прогрессивных потоковых форматов может рассматриваться еще такая характеристика, как битрейт - объем данных, который требуется передать для воспроизведения одной секунды видео.

Проблемы с объемом передачи данных для воспроизведения видео аналогичны с тем, что обсуждалось для аудиофайлов. Для фрагмента, длительность которого ограничивается несколькими секундами, содержащий его файл можно хранить непосредственно на сервере и передавать пользователю по прямой ссылке.

Если длительность фрагмента в районе минуты и больше - то следует использовать потоковую передачу, что может потребовать поддержки отдельного потокового сервера. В подобной ситуации возможно использование внешнего хранения файлов на медиа серверах. В этом случае файл размещается на специальном медиа сервере (например YouTube.com), от службы сопровождения сервера получается ссылка на доступ к ресурсу и, если нужно, фрагмент программного кода, который можно встроить в веб-документ, позволяющий получить из веб-документа доступ к ресурсу и обеспечить выполнение ряда 
условий. В случае использования подобной схемы хранения медиа-сервер при взаимодействии с пользователем анализирует состав программного и аппаратного обеспечения на компьютере пользователя, требуемые параметры воспроизведения и передает данные в подходящем формате, даже если на сервер они помещались в другом формате. Более того, если в процессе воспроизведения условия передачи и воспроизведения файла изменяются, медиа-сервер, используя специальные адаптивные алгоритмы, изменяет параметры передаваемого медиа-потока так, чтобы на стороне пользователя файл воспроизводился с наилучшим возможным качеством.

Таким образом, можно сформулировать следующие рекомендации для представления видеофайлов:

- формат файла-контейнера - MP4, OGG (браузеры, поддерживающие стандарт HTML5 могут их воспроизводить самостоятельно);

- Размер кадра (width $\mathrm{x}$ height) соответствует распространенным соотношениям 4x3, 16х9 и др.

\section{7. Видео 360}

В последнее время появились различные специализированные формы воспроизведения Видео. Заметным явлением стало появление Видео360, когда в видеофайле фиксируется изображение во всех направлениях. Воспроизведение может осуществляться на сферический экран, а пользователь, помещенный внутрь, выбирает направление взгляда самостоятельно. Таким образом, удается повышать уровень ощущения личного присутствия. Пользуясь тем, что угол зрения реального пользователя существенно меньше 360 градусов, были разработаны устройства воспроизведения (виртуальные шлемы) позволяющие получить практически аналогичное ощущение присутствия без создания реальных сферических экранов, причем имеется возможность формировать индивидуальные видеопотоки для каждого глаза пользователя. Файловые контейнеры и кодеки, которые используются для кодирования, не отличаются от тех, которые используются для традиционного видео. Появление нового типа мультимедийного ресурса - видео в формате 360 градусов - повлекло сначала создание специализированных плейеров, позволяющих просматривать соответствующий контент в специализированном приложении, браузере, с использованием специальных гарнитур виртуальной реальности, на мобильных устройствах, затем появление возможности просмотра в универсальных плейерах, указывая в качестве параметра тип ресурса, затем встраивание в файл ресурса мета-данных, позволяющее плейерам автоматически распознавать тип ресурса и формировать элементы управления просмотром, подходящие для соответствующей ситуации

Особенностью использования такого типа ресурса является большая требовательность к пропускной способности коммуникационной среды. При современном уровне развития программных средств медиа-сервер отсылает пользователю полное сферическое изображение, а плейер выбирает для демонстрации некоторый фрагмент (примерно 10\% от площади сферы), определяемый углом зрения и направлением «взгляда» пользователя. Это обстоятельство приводит к тому, что для приемлемого качества отображения видео360 минимальные требования к размеру кадра является 4K (распространенный телевизионный формат FullHD соответствует формата 2K. Формирование подобных медиа-потоков накладывает высокие требования на медиа-сервер (особенно, если ресурс пользуется популярностью), поэтом более предпочтительным представляется хранение ресурсов в формате Видео360 на сторонних стриминговых серверах (таких как YouTube) и использования встраиваемого кода для обращения к серверу.

Таким образом, можно сформулировать следующие рекомендации для представления видеофайлов в формате Видео360:

- формат файла-контейнера - MP4, OGG; 
- paзмер кадра (width $\mathrm{x}$ height) соответствует соотношениям $2 \mathrm{x} 1$, формата не менее $4 \mathrm{~K}$;

- при размещении ресурсов на внешних стриминговых серверах в видеофайл внедряется метаинформация, указывающая, что файл должен воспроизводиться в формате Видео360.

\subsection{D модели, сцены виртуальной и дополненной реальности}

Использование 3D-моделей и интерактивных $3 \mathrm{D}$-сцен для содержательной иллюстрации в документах привело к появлению автономных средств отображения таких объектов, в том числе и через интернет. Однако сложность и разнообразие инструментов создания таких ресурсов затрудняют их широкое использование в качестве элементов мультимедийных коллекций. Необходимость установки специализированного программного обеспечения на компьютер пользователя в общем случае не решает проблему отображения ресурса. Так, созданная в 3DS-max сцена может быть экспортирована в различные форматы (в том числе, и предназначенные для обмена 3Dмоделями), однако при экспорте многие особенности модели будут утеряны, так как специфические модификаторы среды разработки не поддерживаются такими форматами. Более того, при использовании специальных плагинов, особенно коммерческих, воспроизведение сцены на компьютере пользователя может оказаться невозможным. Кроме того, для правильного воспроизведения сцены необходимо сохранить структуру проекта, содержащего для сложных сцен десятки каталогов и сотни, может быть, и тысячи отдельных файлов, причем в проекте могут сохраняться абсолютные пути к используемым ресурсам, так что даже при копировании всего дерева проекта в другое место правильное воспроизведение сцены может нарушиться.

Авторам представляется, что решение задачи полноценного использования мультимедийных ресурсов, типа 3D-моделей и интерактивных $3 \mathrm{D}$-сцен, в качестве элементов мультимедийной коллекции может быть найдено, например, одним из следующих способов.

1. Использование специализированных веб-сервисов, позволяющих автору загрузить свой проект на сервер, при необходимости отредактировать и проверить адекватность его отображения и представить код для его внедрения в веб-страницу (как это сделано, например, на youtube.com). В качестве такого сервиса может, например, выступить сервис SketchFab. Недостатком такого решения можно считать то, что ресурс хранится на внешнем сервисе, и возникновение проблем с аккаунтом владельца может сделать ресурс недоступным.

2. Экспорт проекта в формат WebGL. Последнее время поддержка этого формата браузерами постоянно улучшается, кроме того, имеются среды разработки, позволяющие реализовать или отредактировать 3D-проект непосредственно в формате WebGL. 3D-проект, подготовленный в такой технологии, может быть представлен непосредственно на веб-странице средствами исключительно веб-браузера. В качестве среды разработки, например, можно использовать среду, поддерживаемую сообществом ThreeJS.

\section{9. Использование картографической информации}

В ряде профессиональных дисциплин информация привязывается к существующему или существовавшему ландшафту. Развитие современных картографических систем, размещенных в интернет, позволяет каждому пользователю внедрить небольшой код в веб-документ, который обеспечит встраивание нужного фрагмента карты с набором функциональных элементов, которые могут предоставляться как владельцами картографического сервиса, так и добавляться самим пользователем с использованием открытого API. Подобные сервисы предоставляются как российскими платформами, такими ка Yandex, MaiRu, так и глобальными сервисами, такими как Google, Microsoft, 
Apple и др. Причем для внедрения фрагмента карты в документ требуется указание географических координат и, если карта должна обладать дополнительной функциональностью, JavaScript библиотека, представляемая автором и разработанная на основе открытого API выбранного картографического сервиса. JavaScript библиотека представляет собой текстовый файл, который может храниться как текстовый фрагмент в базе данных сервера журнала (сервера поддержки коллекции)

Таким образом, можно сформулировать следующие рекомендации для представления картографических данных:

- Фрагмент кода, обеспечивающего отображение нужного фрагмента карты (обычно формируется на веб-странице соответствующего картографического сервиса), внедряемый в веб-документ.

- JavaScript библиотека, реализующая необходимую функциональность, разработанная на основе открытого API выбранного картографического сервиса (если карта должна обладать дополнительной функциональностью).

- Формулы и диаграммы.

Наряду с используемыми одиночными количественными данными, часто используются массивы таких данных. В этом случае у авторов возникает необходимость продемонстрировать особенности взаимосвязей между элементами массивов. Одним из распространенных способов достижения этой цели является визуализация данных. Функциональность использующихся в настоящее время в полиграфии предварительно подготовленных растровых изображений графиков и диаграмм представляется недостаточными для онлайн отображения. Для решения этой задачи разрабатывается компонент, позволяющий пользователю ввести табличный массив данных и их описаний и выбрать из палитры стиль отображения. Прототип компонента визуализации количественных данных в настоящий момент проходит тестирование.

Немаловажным и востребованным компонентом научной статьи является наличие математического контента. При включении математической нотации в текст необходимо учитывать два аспекта - это написание формулы и их дальнейшее отображение.

Стандартом де-факто при вводе математических уравнений является TeX, который чаще всего используется в виде своего расширения LaTeX.

Второй стороной использования формул в статье, опубликованной в научном on-line журнале, является их корректное отображение в браузере. Существует несколько подходов к решению этой проблемы:

- отображение формул в виде растрового изображения (GIF или PNG). Такой подход хоть и является наиболее простым, но полностью стирает смысл формулы, исключает возможность ее дальнейшего анализа. Небольшим улучшением ситуации может служить публикация LaTeX-формулы в качестве альтернативного текста к изображению;

- конвертация LaTeX-формулы в векторный формат SVG;

- использование специализированного языка математической разметки MathML. MathML предназначен для облегчения использования и обмена данными математической нотации в интернете (подробнее о MathML cм., например в Wikipedia). MathML является приложением XML и может обрабатываться и отображаться браузером. Однако не все браузеры имеют поддержку MathML, что влечет за собой необходимость использования дополнительных библиотек. Особого внимания заслуживает кросс-браузерная JavaScript библиотека MathJAX;

- актуальным является использование автоматической конвертации из LaTeX в MathML;

- одним из вариантов решения задачи полноценного использования формул в журнале с мультимедиа контентом, на взгляд авторов, может являться следующий ряд подходов: 
- использовать формат TeX/LaTeX для формул;

- рекомендовать авторам статей проводить предварительную подготовку формул к публикации с помощью мощных сторонних инструментов, таких, как Overleaf, Authorea, Papeeria или Lyx;

- отображать формулы в браузере с использованием MathML и в качестве инструмента кросс-браузерного отображения и инструмента автоматической конвертации из LaTeX в MathML можно предложить использовать MathJAX.

В настоящий момент ведется разработка компонента платформы, отвечающего за загрузку и визуализацию формул.

\section{Заключение}

Анализ потребностей инструментов, необходимых для поддержки цифровых мультимедийных коллекций показал, что при современном уровне развития программного обеспечения (серверного и клиентского) возможно создание платформы поддержки цифровых мультимедийных коллекций, обеспечивающей формирование, редактирование и хранение цифровых коллекций при приемлемых требованиях к службе эксплуатационной поддержки, позволяющей предоставлять широкому кругу пользователей доступ к коллекции через интернет при использовании браузера без необходимости установки на клиентский компьютер дополнительного программного обеспечения.

К текущему моменту, разрабатываемая система поддерживает механизмы публикации ограниченного набора компонентов, таких как, единичное изображение, публикация коллекции изображений с визуализацией в варианте слайдера и набора миниатюр, видео и видео 360, публикуемые на сервисе YouTube. Нерешенной остается задача публикации востребованных элементов коллекций, таких как $3 \mathrm{~d}$ модели и элементов научного журнала - графиков и математических формул.

Сложные мультимедиа данные требуют оценки и анализа технологий их создания, хранения и визуализации. Анализ форматов этих данных позволил осуществить выбор технологического стека, библиотек и модулей, на базе которых продолжает разрабатываться информационная система. Развитие дополнительных функциональных возможностей идет в нескольких направлениях

1. Разрабатываются модули публикации 3D моделей и интерактивных 3D схемы на базе сервиса ScetchFab и использования WebGL. Выбор варианта публикации будет зависеть от формата данных, представленных автором.

2. Начата реализация задачи полноценного использования формул в журнале с мультимедиа контентом. Авторам рекомендуется представлять формулы в форматеTeX/LaTeX и проводить их подготовку на одном из внешних ресурсов, таких как Overleaf, Authorea, Papeeria или Lух. Модуль, позволяющий отображать формулы в браузере и автоматически конвертировать из (La)TeX в MathM, будет использовать JavaScript библиотеку MathJAX.

3. Расширен спектр публикуемых мультимедиа данных, которые не требуют дополнительной обработки и могут напрямую отображаться браузерами, а именно, аудио в формате MP3, векторная графика в формате SVG

Информационная система развивается в двух направлениях и реализуется на базе единого ядра с несколько различающейся функциональностью:

- создание и поддержка мультимедиа коллекций;

- онлайн мультимедиа научный журнал.

Комплексный подход в разработке обоих направлений сопровождается созданием инсталляторов и позволяет разворачивать системы из коробки на любом стандартном современном виртуальном хостинге. 
Планируемое развитие направлено на увеличение различных сопровождаемых мультимедиа форматов и разработку модулей экспорта метаданных в ветке электронного журнала.

Авторам элементов коллекции может быть предоставлен инструментарий для загрузки и онлайн редактирования. Поддерживаемый набор форматов данных может обеспечить широкий спектр используемых ресурсов.

Работа проведена в Санкт-Петербургском государственном университете при поддержке проекта РФФИ 19-07-01012 А.

\title{
Литература
}

[1] DSpace: an open source solution for accessing, managing and preserving scholarly works. 2007. URL: http://www.dspace.org/ (дата обращения: 22.11.2019).

[2] Федотов А. М., Байдавлетов А. Т., Жижимов О. Л., Самбетбаева М. А., Федотова О. А. Цифровой репозиторий в научно-образовательной информационной системе // Вестн. Новосиб. гос. ун-та. Серия: Информационные технологии. 2015. Т. 13, вып. 3. C. $68-86$.

[3] Горбунов-Посадов М.M. Живая публикация // ИПМ им. Келдыша PAH. 2011. URL: http://www.keldysh.ru/gorbunov/live.htm (дата обращения: 22.12.2019).

[4] Что такое научное рецензирование? [Электронный pecypc] // Elsevier https://www.elsevier.com/reviewers/what-is-peer-review (дата обращения: 20.11.2019).

[5] М.М.Горбунов-Посадов [и др.]. Анимация и видео в научной публикации // Препринты ИПМ им. М.В.Келдыша. 2014. № 104.32 с. URL: http://library.keldysh.ru/preprint.asp?id=2014-104 (дата обращения: 02.11.2019).

[6] Борисов, Н.В. Проектирование программной платформы полного издательского цикла для издания сетевого мультимедийного журнала / Борисов, Н.В., Захаркина, В.В., Мбого, И.А., Прокудин, Д.Е. // Культура и технологии. 2017. Том 2. С. 21-28. URL: http://cat.ifmo.ru/ru/2017/v2-i1/100 (дата обращения: 29.03.2020).

[7] Борисов Н.В., Захаркина В.В., Мбого И.А., Прокудин Д.Е., Щербаков П.П. Проблемы создания онлайн научного журнала с мультимедиа контентом // Научный сервис в сети Интернет: труды XXI Всероссийской научной конференции (23-28 сентября 2019 г., г. Новороссийск). М.: ИПМ им. М.В.Келдыша, 2019. С. 153-165. URL: http://keldysh.ru/abrau/2019/theses/87.pdf doi:10.20948/abrau-2019-87 (дата обращения: 21.02.2020).

\section{About Choosing Data Formats for Electronic Multimedia Collections}

\author{
N.V. Borisov, V.V. Zakharkina, I.A. Mbogo, P.P. Shcherbakov
}

\section{Saint Petersburg State University}

The system is being developed to ensure full access to multimedia collections via the web interface without installing additional applications or plugins on the client side. One area of the application is electronic scientific journal support.

Requirements dictated by the peculiarities of storage, access and presentation of multimedia elements of electronic collections by support service, content owners and external users are analyzed.

The article describes the reasons for including or not supporting certain data formats of collection elements using the example of results obtained during the creation of an electronic scientific journal tool platform, which provides publication of multimedia materials through the web interface. 
The multimedia collection publishing system, which currently supports a limited set of formats, has been brought to the out-of-the-box deployment level in both the universal and electronic multimedia journal variants. It is planned to further support all the formats discussed in the article.

Keywords: multimedia content, electronic collection, data formats

Reference for citation: Borisov N.V., Zakharkina V.V., Mbogo I.A., Shcherbakov P.P. About Choosing Data Formats for Electronic Multimedia Collections// Information Society: Education, Science, Culture and Technologies of the Future. Vol. 4 (Proceedings of the XXII International JointScientific Conference «Internet and Modern Society», IMS-2020, St. Petersburg, June 17-20, 2020). - St. Petersburg: ITMO University, 2020. P. 58 - 71. DOI: $10.17586 / 2587-8557-2020-4-58-71$

\section{Reference}

[1] DSpace: an open source solution for accessing, managing and preserving scholarly works. [Электронный ресурс] / MIT Libraries; HP Labs. 2007. http://www.dspace.org/ (access date: 20.05 .2020$)$.

[2] Fedotov A. M., Baydavletov A. T., Zhizhimov O. L., Sambetbaeva M. A., Fedotova O. A. Tsifrovoy repozitoriy v nauchno-obrazovatel'noy informatsionnoy sisteme // Vestn. Novosib. gos. un-ta. Seriya: Informatsionnye tekhnologii. 2015. T. 13, vyp. 3. S. 68-86. (In Russian).

[3] Gorbunov-Posadov M.M. Zhivaya publikatsiya [Elektronnyy resurs] // IPM im. Keldysha RAN. 2011, redaktsiya ot 02.10.2018. http://www.keldysh.ru/gorbunov/live.htm (access date: 20.05.2020). (In Russian).

[4] Chto takoe nauchnoe retsenzirovanie? [Elektronnyy resurs] // Elsevier https://www.elsevier.com/reviewers/what-is-peer-review (access date: 20.05.2020).

[5] M.M.Gorbunov-Posadov [i dr.]. Animatsiya i video v nauchnoy publikatsii // Preprinty IPM im. M.V.Keldysha. 2014. No 104. 32 s. URL: http://library.keldysh.ru/preprint.asp?id=2014104 (access date: 20.05.2020). (In Russian).

[6] Borisov, N.V. Proektirovanie programmnoy platformy polnogo izdatel'skogo tsikla dlya izdaniya setevogo mul'timediynogo zhurnala [elektronnyy tekst] / Borisov, N.V., Zakharkina, V.V., Mbogo, I.A., Prokudin, D.E. // Kul'tura i tekhnologii. 2017. Tom 2. S. 21 28. URL: http://cat.ifmo.ru/ru/2017/v2-i1/100 (access date: 20.05.2020). (In Russian).

[7] Borisov N.V., Zakharkina V.V., Mbogo I.A., Prokudin D.E., Shcherbakov P.P. Problemy sozdaniya onlayn nauchnogo zhurnala s mul'timedia kontentom // Nauchnyy servis v seti Internet: trudy XXI Vserossiyskoy nauchnoy konferentsii (23-28 sentyabrya 2019 g., g. Novorossiysk). M.: IPM im. M.V.Keldysha, 2019. S. 153-165. URL: http://keldysh.ru/abrau/ 2019/theses/87.pdf doi:10.20948/abrau-2019-87 (access date: 20.05.2020). (In Russian). 